\title{
Patent foramen ovale closure: To do or not to do!
}

\author{
Mohammad M. Ansari MD, Solomon B. Spiegel DO
}

\begin{abstract}
Patent foramen ovale (PFO) closure is a long-standing topic of debate and discussion, which lends itself to ongoing research into interventions in this area. The reported prevalence of PFO is $\sim 25 \%$ in the population and appears to be more prevalent in younger populations due to closure later in life. Based on imaging and autopsy studies, the prevalence is near $35 \%$ in patients less than 30 years old, $25 \%$ in patients 30 to 80 years old, and $20 \%$ in patients older than 80 years old. ${ }^{1}$ In spite of this high prevalence, many have viewed the PFO as a vestigial structure and in many cases requires no intervention. This structure is formed during fetal life when the septum primum grows from the atrial roof to the endocardial cushions, and the septum secundum grows from the right atrial side toward the endocardial cushion; the overlap formed between the septum secundum and the septum primum forms the fossa ovale. This allows a connection between the left and right atria at the fossa ovale and is essential to normal fetal circulation by allowing oxygenated blood from the umbilical veins entering the heart through the inferior vena cava to be shunted across the atrial septum and bypass the pulmonary circulation. At birth, the fossa ovale fuses in response to the change to respiratory dynamics, and this should lead to closure of the foramen ovale.
\end{abstract}

The defect is usually identified with ultrasoundbased imaging. In many patients, a PFO can be detected with a transthoracic echocardiogram (TTE) with agitated saline. An intra-cardiac shunt is suggested by passage of agitated saline bubbles within three cardiac cycles of right atrial opacification. Valsalva maneuvers performed during the bubble study in an awake patient will increase right atrial

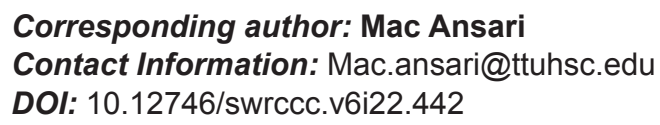

pressure and increase the likelihood of detection of the PFO. The shunts may be quantified based on the number of bubbles seen in the left atrium during the study. Sources vary on the exact number of bubbles, but fewer than 10 is consistent with a small shunt, 10 to 20 bubbles is a moderate shunt, and more than 20 or 25 bubbles is a large shunt. Based on meta-analyses of published data, the sensitivity of TTE for PFO detection is $46 \%$ and the specificity is $99 \% .^{2}$ The transesophageal echocardiogram (TEE) is considered the gold standard study for diagnosis of a PFO with sensitivity of $89 \%$ and specificity of $92 \%{ }^{3}$ Transesophageal echocardiograms provide much better characterization of PFO anatomy but may miss some small PFOs due to the inability of patients to perform Valsalva maneuvers when they are sedated. A third modality, the transcutaneous Doppler, may actually be better than the TEE for diagnosis of a PFO, but it does not offer any anatomical context and cannot distinguish between intracardiac and intrapulmonary shunts. ${ }^{4}$

After a PFO is diagnosed, the decision must be made regarding closure. The crux of the issue is that PFOs are relatively common, but closure is not needed in many patients with nonspecific symptoms. The following clinical conditions have been suggested for PFO closure devices: cryptogenic stroke, migraine, decompression sickness, hypoxia, platypnea-orthodeoxia syndrome, and obstructive sleep apnea. ${ }^{5}$ The most significant of these conditions is the issue of cryptogenic stroke. Many observational studies were initially performed to assess the relationship between PFO and cryptogenic stroke, and there are now four multicenter, randomized control trials that have been published evaluating PFO closure for secondary prevention of stroke: Closure or Medical Therapy for Cryptogenic Stroke with Patent Foramen Ovale (CLOSURE), Percutaneous Closure of Patent Foramen Ovale in Cryptogenic Embolism (PC trial), Closure of Patent Foramen Ovale vs. Medical Therapy 
after Cryptogenic Stroke (RESPECT), and Patent Foramen Ovale Closure or Antiplatelet Therapy for Cryptogenic Stroke (REDUCE). The CLOSURE trial randomized the STARFlex and CardioSEAL closure devices for PFO closure compared with medical therapy (warfarin, aspirin, or both) in 909 patients with cryptogenic stroke or TIA within two years with a primary composite endpoint of stroke, TIA, and death. ${ }^{6}$ In this study, the primary endpoint was present in $5.5 \%$ of the patients receiving the device and $6.8 \%$ of patients receiving medical therapy without significant reduction in the stroke or TIA rates. This study was criticized for using the STARFlex device, which has low rates of procedural success, for including patients with TIA which can be difficult to define, and for not having a long enough follow-up period. In addition, both of these devices are not available in the US market.

The PC Trial was a smaller trial of 414 patients under the age of 60 with cryptogenic stroke using the modern AMPLATZER PFO Occluder compared to medical therapy (with oral anticoagulants followed by oral antiplatelet). ${ }^{7}$ The primary endpoint included peripheral embolism, stroke, TIA, or death from any cause and had a mean follow-up of approximately four years. This study did not demonstrate a significant difference in stroke or TIA or the primary endpoint, but did demonstrate a trend toward benefit of the AMPLATZER Occluder with a reduction in stroke or TIA from $5.2 \%$ in the medical therapy arm to $2.5 \%$ in the device arm. This study suffered from being underpowered and also from a low total event rate of the primary endpoint at follow-up. The RESPECT trial was the next trial published and included 980 patients aged 18 to 60 with cryptogenic stroke to the AMPLATZER PFO Occluder versus medical therapy with either antiplatelet or anticoagulation therapy. ${ }^{8}$ The primary endpoint included stroke and death from any cause. Once again, there was a trend, but no significant difference in favor of device implantation for prevention of stroke. On sub-group analysis, patients with a substantial shunt and presence of an atrial septal aneurysm showed a statistically significant reduction in stroke in the device arm. The REDUCE trial enrolled 664 patients aged 18 to 59 with cryptogenic stroke in the prior 6 months and approximately $80 \%$ with at least a moderate shunt to receive the AMPLATZER PFO Occluder plus antiplatelet therapy versus medical therapy with antiplatelet therapy alone with a primary endpoint of stroke. ${ }^{9}$ The primary endpoint occurred in $1.4 \%$ of the device arm versus $5.4 \%$ in the medical therapy arm for a significant difference. The most significant complication across all trials was an increased rate of atrial fibrillation in patients receiving the device.

Based on these trials and subsequent metaanalysis, the AMPLATZER PFO Occluder (Figure) was approved by the FDA in October 2016 for secondary prevention of stroke in patients with cryptogenic stroke between the ages of 18 and 60. Due to the prevalence of disease and potential for abuse

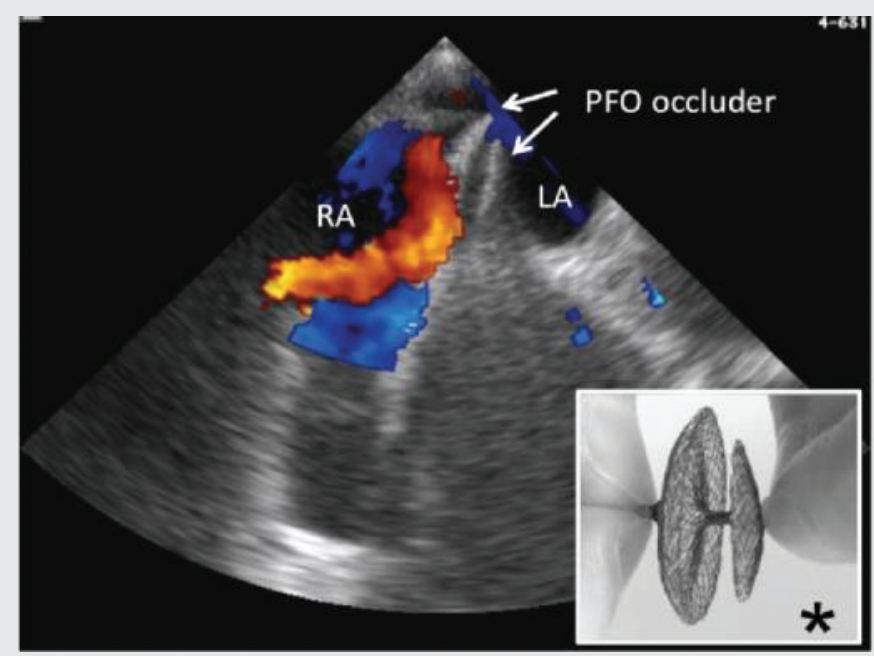

Figure. Transesophageal echocardiography shows the PFO Occluder in the correct position sealing the PFO in the atrial septum. Color Doppler imaging shows a venous blood stream (red) arising from the IVC and rebounding at the right-sided part of the Occluder device. *Image shows a model of the Amplatz PFO Occluder used. LA, left atrium; RA, right atrium. Accessed from TTUHSC library-Open $i$-on 1/10/2018. https://openi.nlm.nih.gov/detailedresult. php?img=PMC3205784_hi-2011-2-e13-g002\&query= patent + foramen + ovale $\& i t=x g \&$ req $=4 \&$ npos $=72$. 
of this device, it was recommended that a neurologist and cardiologist agree on the indication prior to proceeding with implantation. Contraindications to the device include intra-cardiac mass, vegetations, tumor or thrombus at the implant site, anatomical limitations, active endocarditis, untreated infections, or other sources of right-to-left shunts. In addition, patients with nickel allergy may have an allergic reaction to the device that may last up to 60 days after device implantation. Due to the FDA's approval of this device and the latest results, predominantly from the RESPECT and REDUCE trials, there is an expectation that the guidelines for PFO closure will be updated to reflect these data and endorse its use. There remain ongoing off-label uses for PFO closure in decompression sickness, migraines, and platypnea-orthodeoxia syndrome. With regard to decompression sickness, the data seems to favor closure; however, this represents a small patient population of divers suffering from decompression sickness who have also been diagnosed with a PFO. The much larger subset of patients with migraines with aura have less definitive evidence, and the evidence is not strong enough to recommend placement in these patients.

In summary, the AMPLATZER PFO Occluder received FDA approval in 2016 for cryptogenic strokes with a right-to-left shunt in young patients for secondary prevention. There is more evidence of benefit in patients with moderate or large shunt size, atrial septal aneurysm, and with continuation of antiplatelet agents following device implantation. Rates of atrial fibrillation were significantly increased in patients receiving device implantation. The use of long term event monitoring was not routinely performed prior to implantation.

Keywords: patent foramen ovale, right to left shunt, cryptogenic stroke
From: Department of Internal Medicine at Texas Tech University Health Sciences Center, Lubbock, TX

Submitted: $1 / 7 / 2018$

Conflicts of interest: none

\section{REFERENCES}

1. Hagen PT, Sholz DG, Edwards WD. Incidence and size of patent foramen ovale during the first 10 decades of line: an autopsy study of 965 normal hearts. Mayo Clin Proc 1984;59: $17-20$.

2. Mojadidi MK, Winoker JS, Roberts SC, et al. Accuracy of conventional transthoracic echocardiography for the diagnosis of intracardiac right-to-left shunt: a meta-analysis of prospective studies. Echocardiography 2014;31:1036-1048.

3. Mojadidi MK, Bogush N, Caceres JD, et al. Diagnostic accuracy of transesophageal echocardiogram for the detection of patent foramen ovale: a meta-analysis. Echocardiography. 2014; 31:752-758.

4. Mojadidi MK, Roberts SC, Winoker JS, et al. Accuracy of transcranial Doppler for the diagnosis of intracardiac rightto-left shunt: a bivariate meta-analysis of prospective studies. JACC Cardiovasc Imaging 2014; 7:236-250.

5. Dahlöf, CGH, Søndergaard L, Hannam PH. The rationale for PFO closure: a series of arguments for and against. Vascular Disease Management 2009;6(3):80-90.

6. Furlen AJ, Reisman M, Massaro J, et al. Closure or medical therapy for cryptogenic stroke with patent foramen ovale. N Engl J Med 2012;366:991-999.

7. Meier B, Kalesan B, Mattle H, et al. Percutaneous closure of patent foramen ovale in cryptogenic embolism. $N$ Engl J Med 2013;368:1083-1091.

8. Carroll JD, Saver JF, Thaler DE, et al. Closure of patent foramen ovale vs. medical therapy after cryptogenic stroke. $N$ Engl J Med 2013;268:1091-1100.

9. Søndergaard L, Kasner SE, Rhodes JF, et al. Patent foramen ovale closure or antiplatelet therapy for cryptogenic stroke. N Engl J Med 2017;377:1033-1042. 